\title{
Gas Uptake in Solvents Confined in Mesopores: Adsorption versus Enhanced Solubility
}

\author{
Linh Ngoc Ho, ${ }^{\dagger}$ Stéphanie Clauzier, ${ }^{\dagger}$ Yves Schuurman, ${ }^{\dagger}$ David Farrusseng, ${ }^{\dagger}$ and Benoit Coasne ${ }^{*} \neq, \S$ \\ ${ }^{\dagger}$ Institut de Recherches sur la Catalyse et l'Environnement de Lyon (IRCELYON), UMR 5256 CNRS, Université Lyon-1, 2, Avenue \\ Albert Einstein, F-69626 Villeurbanne, France \\ ${ }^{\ddagger}$ Department of Civil and Environmental Engineering, Massachusetts Institute of Technology, 77 Massachusetts Avenue, Cambridge, \\ Massachusetts 02139, United States

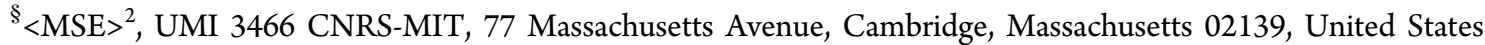

\begin{abstract}
Three molecular mechanisms for gas uptake in a solvent confined in mesopores are identified. On the one hand, $\mathrm{CO}_{2}$ uptake is an adsorption-driven phenomenon that arises from the strong interaction between the gas molecules and the pore surface. On the other hand, $\mathrm{H}_{2}$ uptake is a confinement-induced enhanced solubility in which solubility is favored in the regions of low solvent density formed by the layering of the solvent. In partially filled pores, adsorption at the gas/liquid solvent interface is a third mechanism that leads to large gas uptakes. This study, which sheds light on previously reported yet unclear oversolubility in pores, provides a guide to design hybrid porous catalysts consisting of a solvent confined in a porous solid.
\end{abstract}

G as solubility in solvents, even those confined in macroporous solids, is commonly described using Henry's law which assumes that, in the low-pressure range, the concentration of the dissolved gas is proportional to its partial pressure above the solvent (provided that the solvent does not chemically react with the gas and that the gas partial pressure is low enough). ${ }^{1}$ However, recent studies have reported that, by reducing the solvent volume to the nanometer scale by means of confinement in porous materials, significant gas "oversolubility" can be attained as compared with the values predicted by Henry's law. Luzar and Bratko ${ }^{2,3}$ showed by Molecular Dynamics simulations an increase of $\mathrm{N}_{2}$ and $\mathrm{O}_{2}$ solubility in water by a factor of 5-10 under confinement in hydrophobic nanopores $(38-43 \AA)$. Drastic $\mathrm{H}_{2}$ solubility enhancements were also found for a wide series of solvents $\left(\mathrm{CHCl}_{3}, n\right.$-hexane, water and ethanol) confined in coarsegrained mesoporous $\gamma$-alumina $(d=109 \AA)$ and silica $(d=130$ $\AA)$ and in mesostructured MCM-41 $(d=34 \AA$ A), MCM-41 with $\mathrm{Si} / \mathrm{Al}=1(d=31 \AA)$, SBA-15 $(d=68 \AA)$, and silica aerogel $(d=$ $86 \AA) .{ }^{4-8}$ More recently, we also reported a significant increase of $\mathrm{H}_{2}$ solubility in $n$-hexane and ethanol confined in Cr-MIL101 $(d \sim 30 \AA)$, which was much larger than for MCM-41 silica having a similar pore size. ${ }^{8}$ Ho et al. ${ }^{9-11}$ also showed a 6-fold increase of $\mathrm{CO}_{2}$ solubility in $\mathrm{N}$-methyl-2-pyrrolydone (NMP) confined in MCM-41 $(d=32 \AA)$. Molecular simulations performed by the same authors indicated that the higher $\mathrm{CO}_{2}$ solubility arises from the packing of solvent molecules while $\mathrm{CO}_{2}$ molecules were adsorbed near the pore surface. Finally, Soubeyrand-Lenoir et al. ${ }^{12}$ have recently reported a 5-fold increase of $\mathrm{CO}_{2}$ uptake at 0.2 bar in water confined in $\mathrm{Fe}$ MIL100 ( $d=25-29 \AA)$. Despite the studies above, the physical origin of oversolubility in such hybrid sorbents, i.e., consisting of a solvent confined in porous materials, remains unclear. On the one hand, although the role of the gas/solid interactions seems to be a key parameter, the enhanced solubility is often attributed to the structure of the confined liquid, which leads to surface excess concentrations at mesoconfined gas/liquid interfaces. ${ }^{7}$ On the other hand, oversolubility is also thought to occur because of favorable adsorption at the adsorbent surface. Measuring and predicting the solubility of gases in liquids confined in porous solids (triphasic systems) is crucial for a number of industrial processes. ${ }^{13,14}$ These include heterogeneous catalytic systems (hydrogenation and oxidation reactions), as well as oil and shale gas extraction. ${ }^{15}$ So far, a comprehensive and unifying picture of oversolubility in pores has not been reached. The situation is even more puzzling as oversolubility can stem from different mechanisms depending on the gas and solvent being considered. Among questions that remain to be answered, the role of the balance between the gas/ solvent and the gas/solid interactions in such oversolubility effects has not been considered in detail.

In this Letter, we unravel by means of experiments and molecular simulations the physical origin of the so-called "oversolubility". The atomic mechanisms behind oversolubility in hybrid sorbents are identified by considering gases with different interaction types and strengths, $\mathrm{H}_{2}$ and $\mathrm{CO}_{2}$. Following the work by $\mathrm{Ho}$ et al., ${ }^{10}$ we focus on a simple solvent, octamethylcyclotetrasiloxane (OMCTS), confined in mesoporous MCM-41 silica because of its use as a sorbent for 
adsorption and separation. $\mathrm{CO}_{2}$ uptake is shown to be an adsorption-driven phenomenon, which arises from the strong interaction between the gas molecules and the atoms of the pore surface. In contrast, $\mathrm{H}_{2}$ uptake is a confinement-induced enhanced solubility in which the solubility is favored in the regions of low solvent density formed by the layering of the solvent at the surface.

The experiments for $\mathrm{H}_{2}$ uptake were carried out in our laboratory while the experimental data for $\mathrm{CO}_{2}$ were taken from the literature. ${ }^{11}$ The hybrid sorbents were prepared by filling the pores with the solvent via wet impregnation (see details in the Supporting Information (SI)). The measurements were performed using a volumetric apparatus (PCT Pro Setaram). For comparison, $\mathrm{H}_{2}$ adsorption measurements were also carried out on the bare solid and on the bulk solvent. The gas uptake in the hybrid sorbents was expressed as the number of moles of gas divided by the porous volume of the evacuated solid. We checked using gravimetric measurements that the amount of solvent confined in the porous solid remains constant prior and after evacuation of air as well as after $\mathrm{H}_{2}$ uptake. The model of cylindrical MCM-41 pores used in this work has a diameter $D=34 \AA$ and was generated using the procedure reported in ref 16 . The silica pore is carved out of a block of bulk amorphous silica which was first prepared using a simulated annealing technique. In contrast to previous works in this field, the pore in the present study is of a finite length and opened toward external bulk reservoirs so that adsorption at the gas/liquid interface at the pore opening is taken into account (Kelvin meniscus). While the latter interface has been proposed as a possible origin of oversolubility in pores, no conclusive data have been reported. ${ }^{5,6} \mathrm{H}_{2}$ and OMCTS molecules are treated as single Lennard-Jones spheres, ${ }^{17}$ while $\mathrm{CO}_{2}$ molecules are described using the Elementary Physical Model. ${ }^{18}$ OMCTS is a simple physical solvent that consists of molecules having a nearly spherical shape. As a result, although of limited use for chemical applications, it is often considered as an ideal solvent for fundamental studies in physical chemistry. In particular, its simple shape provides OMCTS physicochemical properties that can be easily modeled with simple available forcefields. $\mathrm{CO}_{2}$ and $\mathrm{H}_{2}$ solubility in the hybrid MCM-41, i.e., the MCM41 pore containing the solvent, was simulated using the Grand Canonical Monte Carlo (GCMC) method. The hybrid sorbent was prepared by filling the pore volume with different solvent loadings; a pore with one adsorbed layer of solvent and a pore fully filled with the solvent $\left(\sim 0.6\right.$ and $3 \mathrm{mmol} / \mathrm{cm}^{3}$, respectively). Molecular configurations of the systems are shown in Figure 1. The $\mathrm{CO}_{2}$ and $\mathrm{H}_{2}$ uptakes at a given pressure

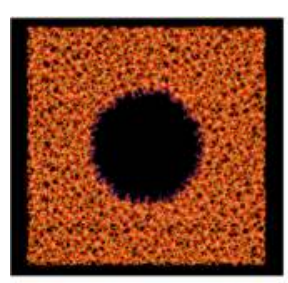

(a)

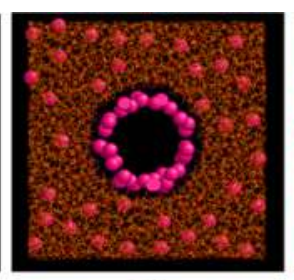

(b)

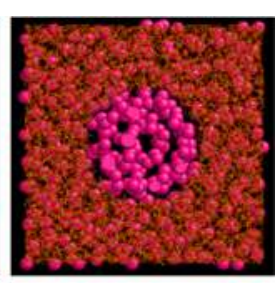

(c)
Figure 1. (a) Bare MCM-41, (b) hybrid MCM-41 with one layer of solvent, and (c) hybrid MCM-41 with full solvent loading. Yellow, red, and white spheres are $\mathrm{Si}, \mathrm{O}$, and $\mathrm{H}$ atoms of the MCM-41. The pink spheres are the solvent molecules. The pore diameter and length are $D$ $=34 \AA$ and $L=100 \AA$. The size of the simulation box is $71.6 \AA \times 71.6$ $\AA \times 179 \AA$. and temperature in the hybrid MCM-41 were calculated in the course of the GCMC simulations as the ensemble average of the number of gas molecules. Details about the methods and models can be found in the SI.

Our models were first validated by comparing the simulated adsorption isotherms of $\mathrm{H}_{2}$ and $\mathrm{CO}_{2}$ with the experimental results for the bulk solvent, the bare MCM-41 solid, and the hybrid MCM-41 (Figure S3 in the SI). For both $\mathrm{CO}_{2}$ and $\mathrm{H}_{2}$, the simulated data are in good agreement with the experimental data (although the simulated data slightly overestimate the $\mathrm{H}_{2}$ amounts). The deviations between the simulated and experimental data for $\mathrm{H}_{2}$ at pressures larger than 3 bar are believed to be due to the use of a simple $\mathrm{H}_{2}$ model with a single LJ center. Despite the small differences observed between the experimental and simulated data for $\mathrm{H}_{2}$ and $\mathrm{CO}_{2}$, the simulations for each system qualitatively reproduce the shape of the experimental isotherms. Therefore, the models used in this work can be considered representative and reliable, as they describe in an adequate fashion both the gas/solvent and gas/ solid interactions.

Figure 2 shows the simulated $\mathrm{CO}_{2}$ and $\mathrm{H}_{2}$ uptakes at $298 \mathrm{~K}$ for pressures up to 10 bar in the bulk solvent and in the hybrid sorbents (completely and partially filled by the solvent). For each gas, Figure 2 also shows the bulk gas density as a function of pressure. Comparison with the experimental data for $\mathrm{CO}_{2}$ and $\mathrm{H}_{2}$ in the bulk solvent and in the hybrid sorbent fully loaded with the solvent shows that our simulations describe quantitatively the gas solubility as well as the gas uptake in the hybrid sorbents. For both gases, an apparent oversolubility is observed since, at a given pressure, the gas uptake in the hybrid sorbents is larger than the bulk solubility. The $\mathrm{CO}_{2}$ and $\mathrm{H}_{2}$ uptakes increase as follows: bulk solvent $<$ hybrid system with full solvent loading < hybrid system with one solvent layer. As will be discussed in more details below, the fact that the largest $\mathrm{H}_{2}$ and $\mathrm{CO}_{2}$ uptakes are obtained for the hybrid sorbent with one solvent layer shows that adsorption at the gas/liquid interface is a major contribution to oversolubility. Due to the presence of the solvent, the maximum $\mathrm{H}_{2}$ and $\mathrm{CO}_{2}$ uptake in the hybrid sorbent decreases as the pore volume occupied by the solvent becomes inaccessible to the gas. It is interesting to compare the $\mathrm{CO}_{2}$ and $\mathrm{H}_{2}$ uptake isotherms for each system (i.e., bulk solvent and hybrid sorbent); $\mathrm{CO}_{2}$ exhibits a greater uptake than $\mathrm{H}_{2}$ due to its stronger interaction with both the silica material and the solvent.

The $\mathrm{H}_{2}$ uptake increases linearly with pressure for all systems, which suggests that the uptake mechanism obeys Henry's law (i.e., solubility). By contrast, the $\mathrm{CO}_{2}$ uptake in the hybrid sorbent follows a Langmuir-type isotherm. These results show that the uptake mechanism for $\mathrm{CO}_{2}$ differs from that for $\mathrm{H}_{2}$ : while $\mathrm{CO}_{2}$ uptake may consist of an adsorption-driven mechanism, $\mathrm{H}_{2}$ uptake may follow a bulk-like solubility mechanism. To verify this hypothesis, we investigated the microscopic mechanisms behind the $\mathrm{CO}_{2}$ and $\mathrm{H}_{2}$ uptakes in the hybrid sorbents. Figure 3 shows the density profiles for $\mathrm{CO}_{2}$, $\mathrm{H}_{2}$, and OMCTS adsorbed at $P=1$ bar in the hybrid sorbent fully filled with OMCTS. For this loading, the solvent forms two layers adsorbed at the pore surface as revealed by the marked density oscillations. A major difference between $\mathrm{CO}_{2}$ and $\mathrm{H}_{2}$ lies in the relative position of the gas and solvent density peaks with respect to the silica surface. Due to the strong interaction between the quadrupole of $\mathrm{CO}_{2}$ and the polar $\mathrm{OH}$ groups at the silica surface, $\mathrm{CO}_{2}$ is preferentially adsorbed at the surface while OMCTS is adsorbed beyond the 

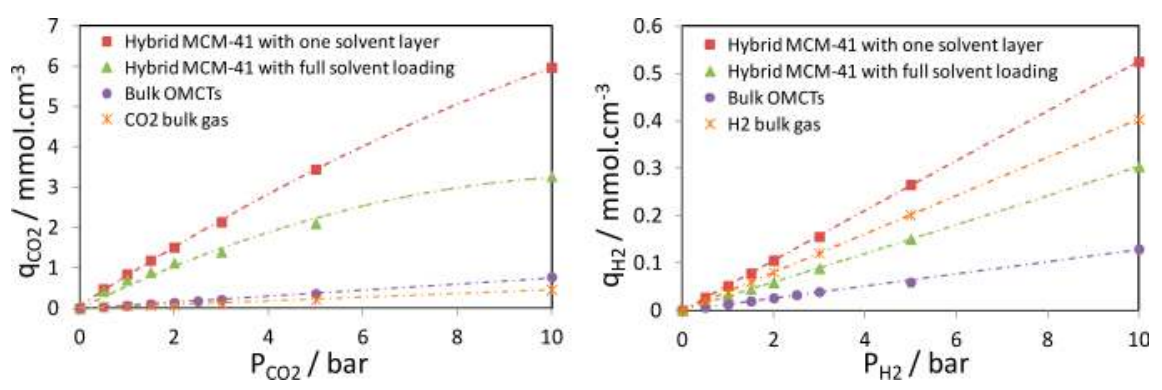

Figure 2. Concentration as a function of pressure for $\mathrm{CO}_{2}$ (left) and $\mathrm{H}_{2}$ (right) in hybrid MCM-41 and in bulk solvent at $T=298 \mathrm{~K}$. The bulk gas density is also shown. The dashed lines are provided as a guide to the eye.
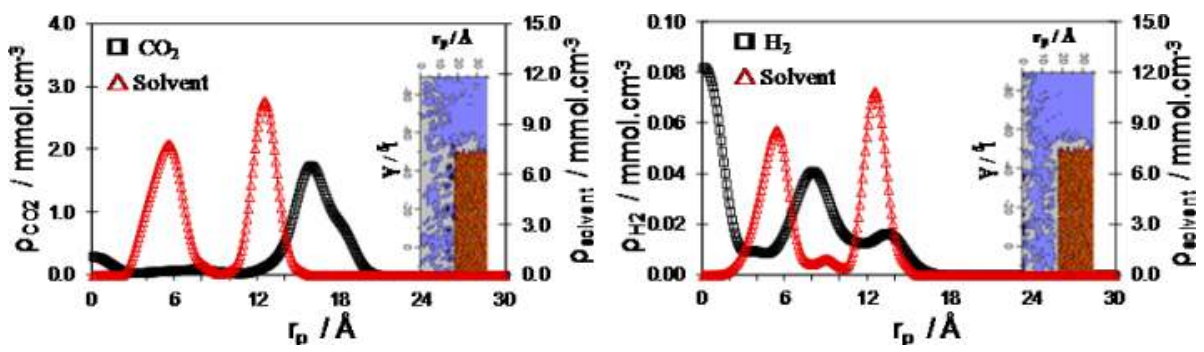

Figure 3. Density profiles for $\mathrm{CO}_{2}$ (left) and $\mathrm{H}_{2}$ (right) in hybrid MCM-41 fully loaded with OMCTS at $P=1$ bar and $T=298 \mathrm{~K}$. The inset shows a contour plot of the density $\rho\left(r_{\mathrm{p}}, z_{\mathrm{p}}\right)$ at a position $r_{\mathrm{p}}, z_{\mathrm{p}}$ within the pore. The pore is shown with the following color code: yellow - silicon, red oxygen, and white - hydrogen.

$\mathrm{CO}_{2}$ layer. On the other hand, since $\mathrm{H}_{2}$ has a small quadrupole and, hence, interacts weakly with silica, OMCTS is adsorbed close to the silica surface while $\mathrm{H}_{2}$ is adsorbed in regions of low solvent density. The insets in Figure 3 show contour plots of the density $\rho\left(r_{\mathrm{p}}, z_{\mathrm{p}}\right)$ of $\mathrm{CO}_{2}$ and $\mathrm{H}_{2}$ at a position $r_{\mathrm{p}}, z_{\mathrm{p}}$ within the pore. These contour plots confirm that the $\mathrm{CO}_{2}$ and $\mathrm{H}_{2}$ uptakes in hybrid sorbents follow different mechanisms. $\mathrm{A} \mathrm{CO}_{2}$ film, whose thickness increases with pressure, is adsorbed at the pore wall. By contrast, $\mathrm{H}_{2}$ is adsorbed in the pore center in regions of low OMCTS density. These results show that the ratio of the solid/gas to the solid/solvent interaction strengths plays a key-role in the oversolubility mechanism. The strong interaction between $\mathrm{CO}_{2}$ and the solid induces the adsorption of $\mathrm{CO}_{2}$ (adsorption effect), while the structure of the solvent molecules in the mesoporous solid governs the $\mathrm{H}_{2}$ uptake (solubility effect). By modifying the solvent/solid interactions (see SI), we further showed that the $\mathrm{H}_{2}$ uptake is nearly independent of the solvent-solid interaction, which reflects the fact that $\mathrm{H}_{2}$ uptake is a solubility mechanism and, hence, not an adsorption effect occurring at the pore surface. The results above show that the surface-solvent and surface-gas interactions are key factors. In fact, the gas uptake is mainly driven by the ratio of these two interactions. If the gas/solid interaction is stronger than the solvent/solid interaction (such as for $\mathrm{CO}_{2}$ in the present work), oversolubility will be an adsorption-driven mechanism. By contrast, if the gas/solid interaction is weaker than the solvent/solid interaction (such as for $\mathrm{H}_{2}$ in the present work), oversolubility will follow a confinement-induced enhanced solubility mechanism. The interpretation above is supported by the heats of adsorption of $\mathrm{CO}_{2}, \mathrm{H}_{2}$ and OMCTS on silica, which provide an estimate of the strength of the different interactions at play: $-\Delta H_{\mathrm{o}}=34 \pm 9$ and $8 \pm 0.5 \mathrm{~kJ} /$ mol for $\mathrm{CO}_{2}$ and $\mathrm{H}_{2}$ on bare MCM-41 and $22 \pm 5 \mathrm{~kJ} / \mathrm{mol}$ for OMCTS on bare MCM-41 (see details in the SI). It should also be noted that, depending on the different interactions mentioned above, the presence of the solvent can be detrimental to the gas uptake as the latter reduces the pore volume accessible to the gas.

To demonstrate that $\mathrm{CO}_{2}$ and $\mathrm{H}_{2}$ uptakes obey adsorption and bulk-like solubility mechanisms, respectively, we estimated the solubility profile $S(r)$ :

$$
S(r)=\frac{\rho_{\text {gas }}(r)}{\rho_{\text {solvent }}(r)}
$$

where $\rho_{\text {gas }}(r)$ and $\rho_{\text {solvent }}(r)$ are the gas and solvent densities in the hybrid sorbent at a distance $r$ from the pore surface. These solubility profiles are shown in Figure 4 together with the
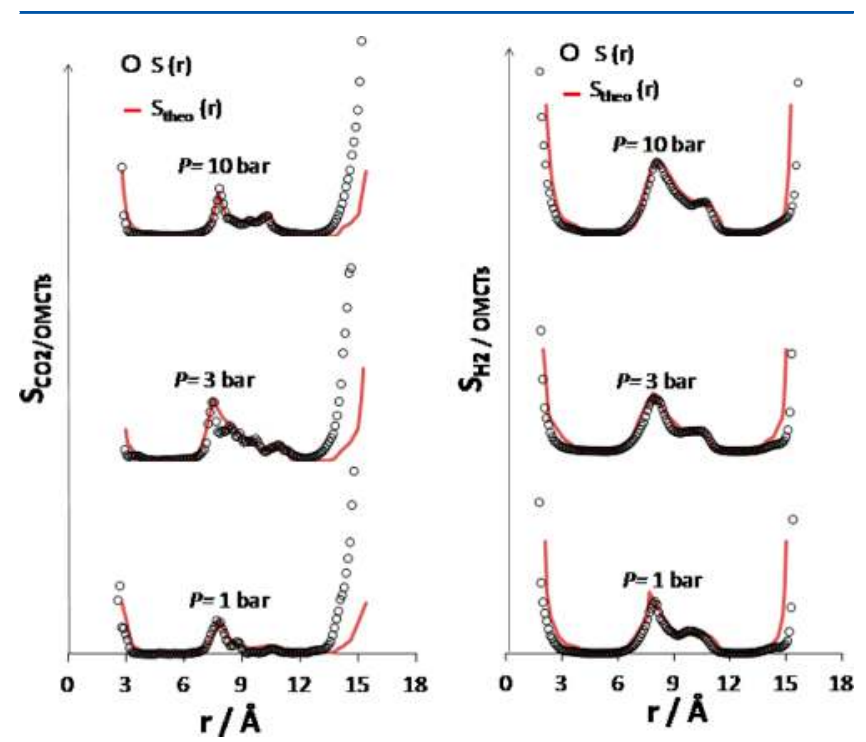

Figure 4. Comparison between the simulated solubility profile $\mathrm{S}(r)$ and the theoretical solubility $S_{\text {theo }}(r)$ for $\mathrm{CO}_{2}$ (left) and $\mathrm{H}_{2}$ (right). $S_{\text {theo }}(r)=S_{\text {bulk }}\left[\rho=\rho_{\text {solvent }}(r)\right]$ where $S_{\text {bulk }}$ is the bulk solubility at a density $\rho$ equal to the local density $\rho_{\text {solvent }}(r)$. 

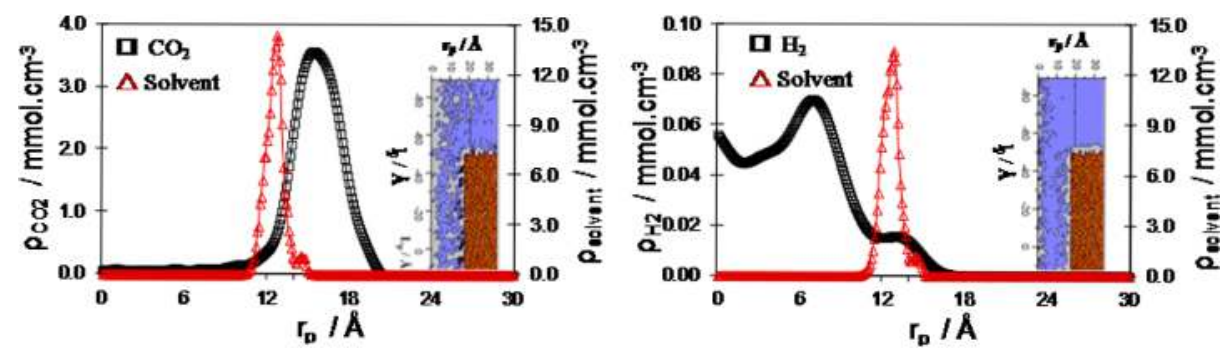

Figure 5. Density profiles for $\mathrm{CO}_{2}$ (left) and $\mathrm{H}_{2}$ (right) in hybrid MCM-41 partially loaded with OMCTS at $P=1$ bar and $T=298 \mathrm{~K}$. The inset shows a contour plot of the density $\rho\left(r_{\mathrm{p}}, z_{\mathrm{p}}\right)$ at a position $r_{\mathrm{p}}, z_{\mathrm{p}}$ within the pore. The pore is shown with the following color code: yellow - silicon, red - oxygen, and white - hydrogen.

solubility profile, $S_{\text {theo }}(r)$, expected on the basis of the local solvent density $\rho_{\text {solvent }}(r)$. For each gas, $S_{\text {theo }}(r)$ was estimated as the bulk solubility $S_{\text {bulk }}$ taken at a solvent density $\rho=\rho_{\text {solvent }}(r)$, i.e., $S_{\text {theo }}(r)=S_{\text {bulk }}\left[\rho=\rho_{\text {solvent }}(r)\right]$. To do that, we estimated the bulk solubility $S_{\text {bulk }}(\rho)$ as a function of $\rho$ by performing Grand Canonical Monte Carlo simulations of $\mathrm{H}_{2}$ and $\mathrm{CO}_{2}$ adsorption in a set of simulation boxes containing the bulk solvent at different densities (see details in the SI). The solubility profile $S(r)$ for $\mathrm{H}_{2}$ is very close to $S_{\text {theo }}(r)$, which demonstrates that $\mathrm{H}_{2}$ uptake in the confined solvent can be described as a bulk-like solubility mechanism. As a result, the gas uptake enhancement observed for $\mathrm{H}_{2}$ in this hybrid sorbent is due to the layering of the confined solvent that creates regions of low solvent density where $\mathrm{H}_{2}$ solubility is favored. Close to the pore center, the solubility profile $S(r)$ for $\mathrm{CO}_{2}$ also follows the expected solubility $S_{\text {theo }}(r)$. This result shows that the $\mathrm{CO}_{2}$ uptake far from the pore surface (i.e., where the interaction potential with silica vanishes) also obeys a solubility mechanism that can be accurately described using the bulk solubility data. By contrast, close to the pore surface, the $\mathrm{CO}_{2}$ uptake in the hybrid sorbent is an adsorption effect driven by the strong interaction between $\mathrm{CO}_{2}$ and silica so that the solubility curve departs from what is expected on the basis of the local solvent density. This demonstrates that the $\mathrm{CO}_{2}$ oversolubility originates from surface adsorption. When adsorption occurs at low pressure in the hybrid sorbent, the $\mathrm{CO}_{2}$ molecules interact with the solid surface so that the highest uptake enhancement is observed. Upon further increasing the pressure, most of the adsorption sites are already occupied so that the additional $\mathrm{CO}_{2}$ molecules cannot easily adsorb as the porosity is filled by the solvent molecules. Finally, despite the oversolubility observed for both $\mathrm{H}_{2}$ and $\mathrm{CO}_{2}$, we would like to emphasize that adsorption in bare MCM-41 is more favorable than oversolubility (in terms of gas amounts that can be stored per unit volume).

It has been proposed that gas adsorption at the gas/liquid interface formed by the solvent and its vapor in porous materials may be the microscopic origin of the so-called "oversolubility". 4 To verify this hypothesis, we now discuss the data obtained when the hybrid solvent contains one solvent layer only. Figure 5 shows the density profiles for $\mathrm{CO}_{2}, \mathrm{H}_{2}$, and OMCTS adsorbed at $P=1$ bar in the hybrid sorbent with one layer of OMCTS. As in the case of the full solvent loading, $\mathrm{CO}_{2}$ is adsorbed close to the pore surface while $\mathrm{H}_{2}$ does not adsorb at the pore surface and is located in the pore volume not occupied by the solvent molecules. Interestingly, for both $\mathrm{CO}_{2}$ and $\mathrm{H}_{2}$, an important gas uptake is observed due to adsorption at the gas/liquid solvent interface formed by the solvent adsorbed film which coexists with the vapor in the pore center.
This result shows that gas adsorption at the gas/liquid solvent interface located within the pore is an important contribution to the oversolubility phenomenon. In particular, this result explains why the $\mathrm{H}_{2}$ and $\mathrm{CO}_{2}$ uptakes at a given pressure are larger for the partial solvent loading than for the full solvent loading. In the case of full solvent loading, gas adsorption at the external gas/liquid interface (Kelvin menisci formed at the pore openings) also contributes to the gas uptake; however, the latter contribution is negligible for most porous materials considered in the literature as their external surface is usually several orders of magnitude lower than their internal surface. In contrast, when the gas/liquid interface is located within the pore (i.e., for partial solvent loadings which are obtained for pressures below the solvent capillary condensation pressure), the gas uptake corresponding to adsorption at the gas/liquid interface is non-negligible as this interface has a surface area of a few square meters per gram.

The findings above shed light on the physical origin of gas enhanced solubility in confined solvents, the so-called "oversolubility", which has been reported in the literature. Depending on the gas and solvent being considered, the gas uptake is either (1) an adsorption-driven effect or (2) a confinementinduced enhanced solubility mechanism (these two mechanisms are illustrated with typical molecular configurations in Figure S5 in the SI). For strong gas/adsorbent interactions, the gas uptake is an adsorption-driven phenomenon that arises from the strong interaction between gas molecules and atoms of the pore surface. By contrast, for weak gas/adsorbent interactions, the gas uptake is a confinement-induced enhanced solubility in which solubility is favored in the regions of low solvent density. In the latter case, the local gas solubility can be described by considering the bulk solubility taken at a density equal to the solvent local density. In partially filled pores, adsorption at the gas/liquid interface also leads to significant gas uptakes (this third oversolubility mechanism is also illustrated in Figure S5 in the SI with a typical molecular configuration). These conclusions provide a guide to design new hybrid adsorbents for gas capture, catalysis, phase separation, etc. The possibility to describe gas uptake in pores using bulk solubility data and adsorption data provides a simple tool to predict and design optimal materials and processes (including the temperature and pressure that should be considered) using simple parameters available in the literature (surface chemistry, pore size, heat of adsorption, adsorbate capacity, solubility, etc.). 


\section{ASSOCIATED CONTENT}

\section{S Supporting Information}

Material preparation, adsorption measurements, and simulation methodology. This material is available free of charge via the Internet at http://pubs.acs.org.

\section{AUTHOR INFORMATION}

\section{Corresponding Author}

E-mail: coasne@mit.edu.

\section{Notes}

The authors declare no competing financial interest.

\section{ACKNOWLEDGMENTS}

We thank Dr. A. Tuel for providing us with MCM samples and Drs. A. Boutin and M. Pera-Titus for helpful comments. This work was supported by OCTAPPOM project in the framework of NEEDS - Nucléaire, Energie, Environnement, Déchets et Société.

\section{REFERENCES}

(1) Henry, W. Experiments on the Quantity of Gases Absorbed by Water, at Different Temperatures, and under Different Pressures. Philos. Trans. R. Soc. London 1803, 93, 29-274.

(2) Luzar, A.; Bratko, D. Gas Solubility in Hydrophobic Confinement. J. Phys. Chem. B 2005, 109, 22545-22552.

(3) Bratko, D.; Luzar, A. Attractive Surface Force in the Presence of Dissolved Gas: A Molecular Approach. Langmuir 2008, 24, 1247-

(4) Miachon, S.; Syakaev, V. V.; Rakhmatullin, A.; M. Pera-Titus, M.; Caldarelli, S.; Dalmon, J.-A. Higher Gas Solubility in Nanoliquids. ChemPhysChem 2008, 9, 78-82.

(5) Pera-Titus, M.; El-Chahal, R.; Rakotovao, V.; Miachon, S.; Dalmon, J.-A. Direct Volumetric Measurement of Gas Oversolubility in Nanoliquids: Beyond Henry's Law. ChemPhysChem 2009, 10, 2082-2089.

(6) Pera-Titus, M.; Miachon, S.; Dalmon, J.-A. Increased Gas Solubility in Nanoliquids: Improved Performance in Interfacial Catalytic Membrane Contactors. AIChE J. 2009, 55, 434-441.

(7) Rakotovao, V.; Ammar, R.; Miachon, S.; Pera-Titus, M. Influence of the Mesoconfining Solid on Gas Oversolubility in Nanoliquids. Chem. Phys. Lett. 2010, 485, 299-303.

(8) Clauzier, S.; Ho, L. N.; Pera-Titus, M.; Coasne, B.; Farrusseng, D. Enhanced $\mathrm{H}_{2}$ Uptake in Solvents Confined in Mesoporous MetalOrganic Framework. J. Am. Chem. Soc. 2012, 134, 17369-17371.

(9) Ho, N. L.; Porcheron, F.; Pellenq, R.J.-M. Experimental and Molecular Simulation Investigation of Enhanced $\mathrm{CO}_{2}$ Solubility in Hybrid Adsorbents. Langmuir 2010, 26, 13287-13296.

(10) Ho, N. L.; Perez, P. J.; Porcheron, F.; Pellenq, R.J.-M. Enhanced $\mathrm{CO}_{2}$ Solubility in Hybrid MCM-41: Molecular Simulations and Experiments. Langmuir 2011, 27, 8187-8197.

(11) Ho, N. L.; Perez, P. J.; Porcheron, F.; Pellenq, R.J.-M. Enhanced $\mathrm{CO}_{2}$ Solubility in Hybrid Adsorbents: Optimization of Solid Support and Solvent Properties for $\mathrm{CO}_{2}$ Capture. J. Phys. Chem. C 2012, 116, 3600-3607.

(12) Soubeyrand-Lenoir, E.; Vagner, C.; Yoon, J. W.; Bazin, P.; Ragon, F.; Hwang, Y. K.; Serre, C.; Chang, J.-S.; Llewellyn, P. L. How Water Fosters a Remarkable 5-Fold Increase in Low-Pressure $\mathrm{CO}_{2}$ Uptake within Mesoporous MIL-100(Fe). J. Am. Chem. Soc. 2012, 134, 10174-10181.

(13) Carberry, J. J. Chemical and Catalytic Reaction Engineering; Dover Publications; Inc.: New York, 2001.

(14) Xu, X. C.; Song, C. S.; Andresen, J. M.; Miller, B. G.; Scaroni, A. W. Novel Polyethyleneimine-Modified Mesoporous Molecular Sieve of MCM-41 Type As High-Capacity Adsorbent or $\mathrm{CO}_{2}$ Capture. Energy Fuels 2002, 16, 1463-1469.
(15) Beenackers, A. A. C. M.; Ertl, G.; Knözinger, H.; Weitkamp, J. In Handbook of Heterogeneous Catalysis; Wiley-VCH, Inc.: Weinheim, Germany, 1997; pp 1444-1464.

(16) Siboulet, B.; Coasne, B.; Dufreche, J.-F.; Turq, P. Hydrophobic Transition in Porous Amorphous Silica. J. Phys. Chem. B 2011, 115, 7881-7886.

(17) Ayappa, K. G.; Mishra; Ratan, K. Freezing of Fluids Confined between Mica Surfaces. J. Phys. Chem. B 2007, 111, 14299-14310.

(18) Harris, J.; Yung, K. H. Carbon Dioxide's Liquid-Vapor Coexistence Curve and Critical Properties As Predicted by a Simple Molecular Model. J. Phys. Chem. 1995, 99, 12021-12024. 